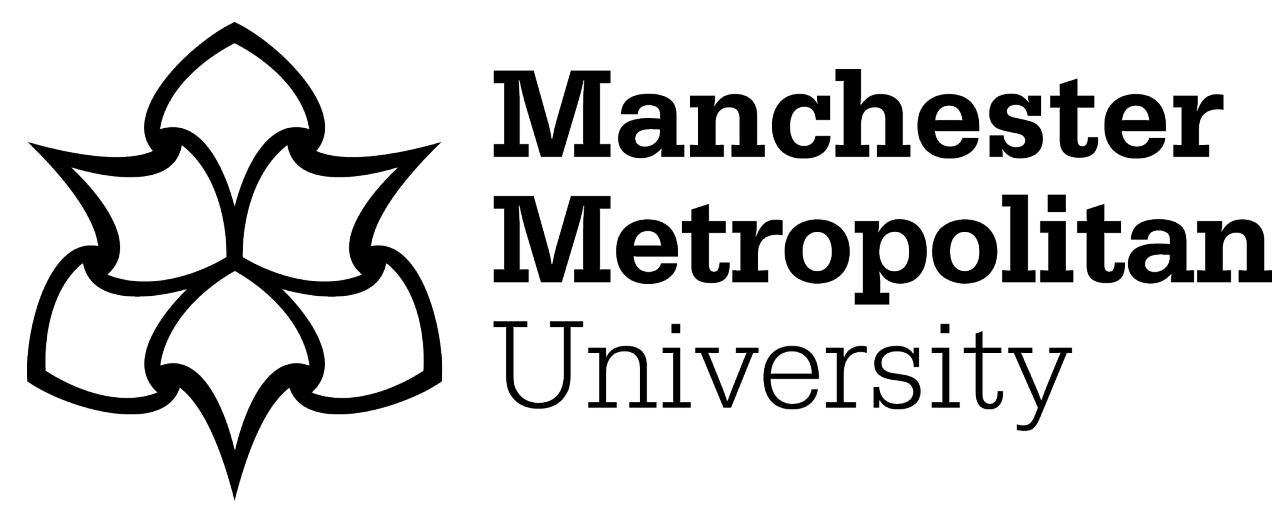

Vafadarnikjoo, Amin ORCID logoORCID: https://orcid.org/0000-0003-21476043, Badri Ahmadi, Hadi, Liou, James JH, Botelho, Tiago and Chalvatzis, Konstantinos (2021) Analyzing blockchain adoption barriers in manufacturing supply chains by the neutrosophic analytic hierarchy process. Annals of Operations Research. ISSN 0254-5330

Downloaded from: https://e-space.mmu.ac.uk/627497/

Version: Published Version

Publisher: Springer (part of Springer Nature)

DOI: https://doi.org/10.1007/s10479-021-04048-6

Please cite the published version 


\title{
Analyzing blockchain adoption barriers in manufacturing supply chains by the neutrosophic analytic hierarchy process
}

\author{
Amin Vafadarnikjoo ${ }^{1}$ (D) . Hadi Badri Ahmadi ${ }^{2}$ James J. H. Liou² ${ }^{2} \cdot$ Tiago Botelho $^{3}$ (D) \\ Konstantinos Chalvatzis ${ }^{3,4}$
}

Accepted: 20 March 2021

(c) The Author(s), under exclusive licence to Springer Science+Business Media, LLC, part of Springer Nature 2021

\begin{abstract}
Tools established for managing information flow in supply chain management and logistics should match digital transformations. This issue is particularly salient for developing nations that hope to achieve sustainable development goals in a globalized era. Modern technologies are required to ensure a secure, transparent, and traceable path of information flow in global supply chains; however, it is not always straightforward for businesses in developing economies to adopt new digital technologies while sustaining productivity. One of the foundational technologies that can be used to create a basis for economic and social systems and to affect manufacturing supply chains in developing economies is blockchain. In this study, we analyze the barriers to blockchain technology adoption in manufacturing supply chains using the neutrosophic analytic hierarchy process (N-AHP). We propose an action plan framework for the validation of blockchain technology in a developing economy. The findings demonstrate that "transaction-level uncertainties" comprise the most critical barrier and have the highest weight in the final ranking followed by "usage in the underground economy", "managerial commitment", "challenges in scalability", and "privacy risks". This paper can assist industrial managers and experts in emerging economies to more clearly identify barriers to the implementation of blockchain technology and show them how to successfully employ blockchain technology in their supply chains.
\end{abstract}

Keywords AHP · Blockchain · Neutrosophic set theory $\cdot$ Supply chain management · Barriers

James J. H. Liou

jamesjhliou@gmail.com

1 Department of Operations, Technology, Events, and Hospitality Management (OTEHM), Business School, Faculty of Business and Law, Manchester Metropolitan University, Manchester M15 6BH, UK

2 Department of Industrial Engineering and Management, National Taipei University of Technology, Taipei, Taiwan

3 Norwich Business School, University of East Anglia, Norwich NR4 7TJ, UK

4 Tyndall Centre for Climate Change Research, University of East Anglia, Norwich NR4 7TJ, UK 


\section{Introduction}

Global supply chain (SC) configurations are becoming increasingly complex and are including numerous international partners. Billions of products are being manufactured, shipped, and delivered to customers through sophisticated SCs involving substantial volumes of data and information (Abeyratne \& Monfared, 2016). Secure information sharing and verifiability are critical, requiring reliable technologies that can trace SCs (Saberi et al., 2019a, 2019b). Digitalization and Industry 4.0 technologies have had a disruptive transformation effect across industries (Ivanov et al., 2019). Although organizations are becoming more informed, the role of digital SCs (DSCs) in adding value as well as the innumerable benefits of digitalization to the SC are not yet fully appreciated (Büyüközkan \& Göçer, 2018). Reaping the benefits of new technologies in SCs is not possible without an appropriate implementation process, which requires a thorough preadoption analysis, including barrier identification. Advances in digital transformation have made numerous opportunities available for optimizing SCs. One of the technologies that could play a central role in shaping future DSCs is blockchain, an emerging technology that offers verified transaction data storage (Petersen et al., 2018). One reason for the rapid growth of blockchain implementation in operations and supply chain management (OSCM) is its capability to provide a solution for the issue of managing sophisticated SCs at a time when transparency, speed, and agility are of the highest importance (Cole et al., 2019). Cole et al. (2019) noted a significant gap in the OSCM literature related to exploring the challenges and opportunities that blockchain offers to the OSCM field.

A blockchain is structured as a "chain" of interconnected data blocks (Babich \& Hilary, 2020). Each transaction made by any member of the SC is time-stamped in the blockchain and recorded in a shared ledger through a decentralized network of computers. This ledger is continuously updated in real-time. New avenues of development have been opened up by foundational technologies such as blockchain, which can also offer visibility to higher tiers in advanced SCs (Babich \& Hilary, 2020). A survey in the World Economic Forum showed that $10 \%$ of global GDP will be stored on blockchains by 2027 (Carson et al., 2018).

White (2017) noted that blockchain research is still in its infancy. Risius and Spohrer (2017) suggested that future studies should emphasize its impact on employment, value creation, and governance rather than technical questions related to design and features. They also indicated that practical contributions to blockchain research are few and far between and focused on only a few topics. Kouhizadeh et al. (2019), Kouhizadeh et al. (2019)) indicated that manufacturing is the field with the highest capability and potential to enhance business value by implementing blockchain technology. In general, real industrial applications of blockchain are inadequate (Pournader et al., 2020). Only a few instances of large-scale blockchain technology have been successfully implemented (Babich \& Hilary, 2020). Carson et al. (2018) determined six unique categories of blockchain utilization and identified them as having two major functions: record keeping and transacting. Overall, the future of blockchain technology seems promising as ever more successful applications are implemented. Currently, blockchain is being integrated with Internet of Things (IoT) sensors to provide integrated cold chains for organs, drugs, and blood (Chanson et al., 2019; Choi et al., 2019).

However, blockchain technology is an unfamiliar area for many SC managers in businesses operating in developing economies. This point is crucial when faced with dramatic organizational change. Entrenched mindsets and managerial practices currently in use can make the process difficult and lengthy. In the next decade, developing 
economies will have to keep up with modern technologies such as blockchain to be able to participate in global SCs, stabilize their positions, and gain competitive advantages for sustainable economic development. Blockchain technology will radically change various aspects of SCs (Dolgui et al., 2020). Naturally, barriers to the implementation of blockchain technology exist that require new adaptations. It is imperative to understand the major barriers that can hinder the appropriate implementation of blockchain technology in SCs in developing economies and what policies should be established to overcome them. White (2017) identified areas in which blockchain technology could have a considerable effect on business. His findings indicated that the usefulness of employing blockchain in global SCM would be uncertain. Extended academic and practical attention is required to realize the benefits of and obstacles to the adoption and development of this system. Frizzo-Barker et al. (2020) conducted a systematic review of research focused on blockchain in the business literature and highlighted the gap and necessity of further research on the application of blockchains in non-Western contexts to understand its socioeconomic impact. Lim et al. (2021) also reviewed the literature on blockchain technology applications in supply chains and revealed research opportunities for using multicriteria decision analysis (MCDA) along with simulation and mathematical modeling.

Thus, a developing economy (i.e., Iran) has been considered as a case in our paper with a particular focus on manufacturing SCs. This study aims to answer the following research questions:

(1) What are the critical barriers to blockchain implementation in a developing economy?

(2) How are these barriers prioritized in manufacturing supply chains in a developing economy?

(3) How can critical barriers assist in validating blockchain implementation in a developing economy?

This study has focused on two aims: first, identifying the most critical barriers to blockchain adoption in manufacturing SCs in a developing economy, and second, to propose an action plan framework for blockchain validation in a developing economy. This paper contributes to the literature on OSCM and MCDA by:

(1) Proposing a multiple-criteria framework for evaluating barriers to blockchain technology in the context of a developing economy;

(2) Integrating neutrosophic set theory (NST) with the analytic hierarchy process (AHP) (i.e., N-AHP) to produce a novel MCDA method for empirically identifying the importance of barriers to blockchain technology;

(3) Proposing an action plan framework for blockchain technology validation in a developing economy.

The rest of this paper is structured as follows. Section 2 presents an overview of the relevant literature. The applied research methodology is presented in Sect. 3. Realworld applications and data analysis are given in Sect. 4. Section 5 presents the analytical results. A discussion including theoretical contributions, theoretical implications, practical implications, and limitations is presented in Sect. 6. Finally, Sect. 7 provides the conclusion. 


\section{Literature review}

This section focuses on the theoretical background of information technology (IT), information systems (IS), and information and communications technology (ICT) in OSCM. Then, some studies on general blockchain technology, blockchain technology in OSCM, and blockchain adoption barriers in SCs are reviewed.

\subsection{Theoretical background}

Rossi et al. (2019) reviewed the research on blockchain in IS. The value and benefits of IT and the use of IS in SCs are significant and well recognized in the literature, particularly for improving supply chain performance (Sharif et al., 2007). Most of the IT-based benefits in SCM arise from the merits of improved information systems by speeding up decisionmaking, raising the visibility of value chain enablers, efficiently meeting expectations of customers, lowering costs of processes, and improving managerial control (Sharif et al., 2007). Lee et al. (2011) suggested that innovative applications of IT will result in value generation for customers, enhanced care, service delivery efficiency, and increased quality of care. Bloom et al. (2014) indicated that in theory, IT is a decentralizing force, while communication technology is a centralizing force. ICT can offer fast, convenient, and inexpensive means of communication, although a gap exists in knowledge about the impact of ICT investments on performance in developed and developing economies. Grant and Yeo (2018) argued that some studies show that ICT investment would not impact performance, whereas others showed the opposite. This relationship becomes more interesting given the context of the study, whether for a developed or developing economy. In one study, the ICT effect on manufacturing efficiency was explored at the firm level in a developing economy (i.e., Tunisia). The results revealed the presence of positive returns to ICT investment (Ayed Mouelhi, 2009).

Blockchain technology is a transformative ICT that has the potential for wide-ranging applications in management, governance, and policy-making (Lin et al., 2017). In a developing economy, it is critical to identify the barriers to blockchain adoption; an action plan framework for blockchain validation can assist in filling this gap, as was highlighted by Frizzo-Barker et al. (2020) in their research on blockchain in non-Western contexts.

\subsection{Blockchain technology}

Blockchain technology is one of the so-called Industry 4.0 technologies among big data, additive manufacturing, industrial cyber-physical systems, and cloud and edge computing (Fernandez-Carames \& Fraga-Lamas, 2019). It is an open, distributed ledger technology (DLT) that can efficiently record digital events such as contracts between participating agents through a nonlocalized, secure, auditable network with smart execution (Iansiti \& Lakhani, 2017). Distributed ledgers are databases that are replicated on all the nodes in the system. In actuality, blockchain requires the utilization of a group of technologies to develop and maintain this type of database. Blockchain technology is currently recognized as the most developed DLT, including a comparison with the directed acyclic graph. One essential advantage of distributed ledgers is that the system is robust even with the failure of single nodes. By contrast, a centralized classical database, where only one master copy of the database exists at any particular time, is more prone to security risks and failure (Babich \& Hilary, 2020). Centralized and distributed or peer-to-peer (P2P) networks are 
illustrated in Fig. 1. The key features of blockchains are decentralization, resiliency, durability, transparency, immutability, authenticity, and speed (Asadi Bagloee et al., 2019). For a discussion on why blockchains are preferable to a centralized ledger, refer to Angelis and Ribeiro da Silva (2019).

Blockchain is the primary technology underlying Bitcoin (i.e., a cryptocurrency) (Andersen \& Ingram Bogusz, 2019; Fosso Wamba et al., 2020); however, there are prototype blockchain applications other than Bitcoin in the IoT, including smart contracts (De Giovanni, 2020), smart property, digital content distribution, Botnet, and P2P broadcast protocols (Yli-Huumo et al., 2016). Blockchain technology also has the capability to alter natural resource use and recycling management (Saberi et al., 2018). Yli-Huumo et al. (2016) conducted a review of the blockchain literature and realized that only $20 \%$ of studies (out of 41 selected papers) addressed blockchain applications, including smart contracts and licensing. Furthermore, they indicated that security and privacy issues have been a central research topic and that most studies have concentrated on improving the current limitations in blockchain technology. Fernández-Caramés and Fraga-Lamas (2019) examined the benefits and challenges of applying blockchains to develop Industry 4.0 and reviewed the latest blockchain-based applications.

\subsection{Blockchain technology in OSCM}

Helo and Hao (2019) indicated that sustainable SCs (Kouhizadeh et al., 2021), safety issues, IoT-based smart assets, and intellectual property rights are the four application areas of blockchains in SCM. Babich and Hilary (2020) identified and explained five key advantages of utilizing blockchain technologies in operations management (OM): visibility, aggregation, validation, automation, and resiliency. Petersen et al. (2018) asked 152 participants working in SC and logistics-related consulting firms or SC and logistics departments in retail or manufacturing companies about their companies' point of view toward blockchain. Their results showed that among SC and logistics companies, nearly $65 \%$ declared that they had observed blockchain development from a distance. They also asked the participants about potential barriers to blockchain adoption in SCs. The answers revealed that regulatory uncertainty was the most critical barrier, and dependence on

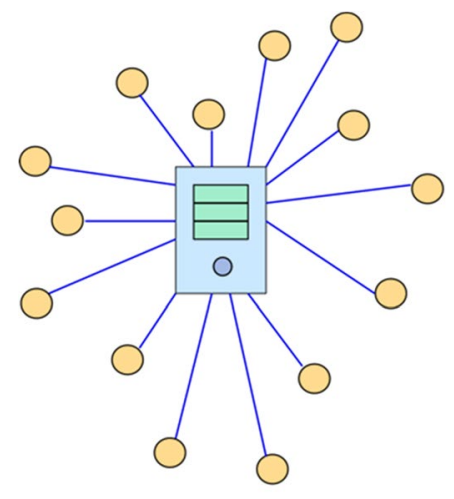

Centralized

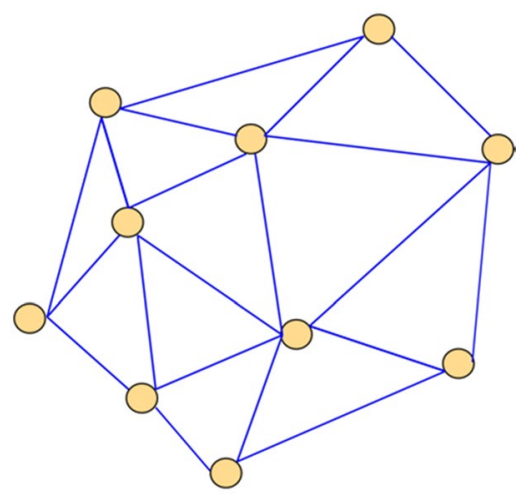

Peer to Peer (P2P)

Fig. 1 Centralized and peer-to-peer networks 
blockchain operators was the least critical barrier. Table 1 presents a summarized overview of recent blockchain technology in OSCM.

\subsection{Blockchain adoption barriers in SCs}

Biswas \& Gupta (2019) explored the barriers to the adoption and implementation of blockchain technology across the industry and service sectors using the DEMATEL method. Wang, Han, et al. (2019) categorized the challenges to blockchain technology diffusion within SCs into three main types: (1) organizational and user-related; (2) technological; and (3) operational. Ozturk and Yildizbasi (2020) divided the barriers to the implementation of blockchain technology in SCs into four types: (1) technological and security; (2) financial and human resources; (3) organization and individual; and (4) social and environmental. Ghode et al. (2020) identified interorganizational trust, interoperability, relational governance, data transparency, data immutability, behavioral intention, product type, and social influence as the most critical challenges, and rated them in the order of importance for the implementation of blockchain technology in SCs. The potential barriers to blockchain adoption that have been identified based on the SCM literature are summarized in Table 2.

\section{Methodology}

The steps in the research methodology and procedure for the applied method are explained below.

\subsection{Research steps}

Steps in the research methodology are illustrated in Fig. 2.

The research method applied in this study is a new development of a popular MCDA method (i.e., AHP) in an uncertain neutrosophic decision-making environment. The proposed N-AHP approach integrates both NST and AHP, with the single-valued trapezoidal neutrosophic numbers (SVTNNs) utilized in the AHP calculations for the first time. The subtraction, division, and inverse operators of the SVTNNs are also revised and adjusted to facilitate the neutrosophic rating scale in the AHP calculations (see "Appendix" for basic definitions of the NST). In other words, the difference between the traditional AHP and the proposed N-AHP lies in the usage of the SVTNNs in the N-AHP instead of the crisp numbers used in traditional AHP. This would enhance the strength of the AHP method in capturing the subjective uncertainty of the involved experts (see Sect. 6.1 for more on the theoretical contributions of the proposed N-AHP).

\subsection{The N-AHP procedure}

The proposed N-AHP method follows the steps below to analyze the identified blockchain barriers and provide a ranking.

Step 1. Decomposing the problem into a hierarchy: 
Table 1 Recent research on blockchain technology in OSCM

\begin{tabular}{ll}
\hline Reference & Characteristics \\
\hline Lim et al. (2021) & $\begin{array}{c}\text { Reviewed the literature on blockchain technology applications in supply } \\
\text { chains }\end{array}$
\end{tabular}

Babich and Hilary (2020)

Bai and Sarkis (2020)

Discussed five major advantages and disadvantages of utilizing blockchain technology in OM

Proposed a group decision-making model which integrated hesitant fuzzy sets and regret theory to evaluate transparency and sustainability for blockchain technology

Chang et al. (2020)

Provided an outline of the latest developments in blockchain technology in global SCM from a variety of sources

Choi (2020)

Choi et al. (2020)

Choi et al. (2020b)

Choi et al. (2020c)

Studied financing problems within SCs for fashionable products by developing an analytical model for blockchain-supported and conventional SCs

Explored product information disclosure on two blockchain-based rental service platforms, applying the Nash game

Explored how blockchain technology can increase the social media analytics utilization for managing supply chain operations

Explored the impacts of customers' risk attitude toward the optimal on-demand service pricing decision by applying the mean-risk theory and identified how the blockchain assists the platform in assessing the proportion of various customers' risk attitudes

Dutta et al. (2020)

Ghode et al. (2020)

Hastig and Sodhi (2020)

Kayikci et al. (2020)

Lohmer et al. (2020)

Manupati et al. (2020)

Pournader et al. (2020)

Rahmanzadeh et al. (2020)

Wamba and Queiroz (2020)

Wong et al. (2020)

Reviewed blockchain literature in relation to supply chain operations and discussed applications, challenges, and future research directions

Prioritized challenges for adoptability of blockchain technology in SCs using gray relational analysis (GRA) and categorized challenges into four groups, i.e., organizational, technological, operational, and social

Presented business requirements and critical success factors for better utilization of supply chain traceability systems

Explored the impact of blockchain technology in food supply chains

Studied resilience in supply chains through blockchain coordination

Applied blockchain technology to monitor SC performance and optimize emissions and operational costs

Conducted a systematic literature review and identified four major clusters in blockchain literature

Applied a fuzzy mathematical model within a blockchain platform to optimize tactical decisions regarding SC objectives and open innovations

Proposed a three-stage model of blockchain adoption in supply chains

Studied the behavioral aspect of blockchain technology adoption in supply chain management

Yoon et al. (2020)

Azzi et al. (2019)

Biswas and Gupta (2019)

Helo and Hao (2019)

Kouhizadeh et al. (2019), Kouhizadeh et al. (2019)

Studied the impact of blockchain technology implementation on firms' exporting performance

Explored how SCs can benefit from blockchains by creating a transparent, reliable, and secure system

Investigated obstacles to blockchain technology adoption and implementation across the industrial and service sectors using DEMATEL

Outlined potential applications of blockchain technology in OM and SCM. Additionally, implemented a blockchain-based logistics monitoring system and tested it based on Ethereum

Conceptualized the links between blockchain technology, product deletion, and the circular economy 
Table 1 (continued)

\begin{tabular}{ll}
\hline Reference & Characteristics \\
\hline Saberi et al. (2019a, b) & $\begin{array}{c}\text { Investigated perceived motives and obstacles for the adoption of } \\
\text { blockchain technology from the perspective of various companies and } \\
\text { industries } \\
\text { Utilized sensemaking theory to determine how emerging blockchain } \\
\text { technology would transform SCs }\end{array}$ \\
Kouhizadeh and Sarkis (2018) & $\begin{array}{l}\text { Reviewed the employment of blockchain technology in green SCs } \\
\text { Reviewed blockchain applications in the literature in terms of informa- } \\
\text { Petersen et al. (2018) }\end{array}$ \\
tion, material, and financial flows in the SC and logistics fields \\
Conducted a structured literature review of the blockchain concept and \\
identified business areas in which blockchain technology could have \\
a considerable effect. Additionally, identified potential future research \\
and development of blockchain technology \\
Reviewed some of the applications of blockchain technology and dis- \\
cussed their potential benefits in manufacturing SCs. Further discussed \\
blockchain adoption challenges in future manufacturing systems
\end{tabular}

To make the problem more comprehensible it is essential to establish a hierarchy representing the goal, criteria, and alternatives in the AHP method. In this study, the decision-making hierarchy includes one level of barriers.

Step 2. Constructing a pairwise comparison matrix:

The experts assess elements (i.e., criteria), based on the relative significance of each element $C_{i}$ over $C_{j}$ using the Saaty importance scale (Saaty, 1980). In the questionnaire, experts choose a linguistic phrase representing the degree of importance of each element in comparison to others. Then, the linguistic phrase is replaced with its corresponding numerical value (i.e., 1 to 9 ).

Given $C_{1}, C_{2}, \ldots, C_{n}$, which signifies the elements, and $a_{i j k}$, which shows a quantified evaluation of a pair of $C_{i}$ and $C_{j}$ elements by the $k^{\text {th }}$ decision maker $(k=1,2, \ldots, p)$. This leads to a pairwise comparison matrix, as represented in Eq. (1) (Hayaty et al., 2014).

$$
A_{k}=\left[a_{i j k}\right]=\left[\begin{array}{cccc}
1 & a_{12 k} & \cdots & a_{1 n k} \\
1 / a_{12 k} & 1 & \cdots & a_{2 n k} \\
\vdots & \vdots & \ddots & \vdots \\
1 / a_{1 n k} & 1 / a_{2 n k} & \cdots & 1
\end{array}\right]
$$

Step 3. Calculating the consistency ratio $(C R)$ :

Saaty (1980) suggested using a consistency test to assess the consistency of the evaluations. The cardinal and output-based consistency in pairwise comparisons can be tested by calculating a CR value, as shown in Eq. (2), where the random index (RI) relies on the number of elements being assessed $(n)$, and $\lambda_{\max }$ is the maximum value of the eigenvector. If $C R \geq 0.1$, then the expert is required to revise their evaluation.

$$
C R=\frac{\left(\lambda_{\max }-n\right)}{(n-1)} / R I
$$

Step 4. Replacing the linguistic information with the SVTNNs: 
Table 2 Barriers to the adoption of blockchain technology in SCs

\begin{tabular}{|c|c|}
\hline Barriers & References \\
\hline Access & Saberi et al. (2019a, b) \\
\hline Challenges in scalability & Biswas \& Gupta (2019) \\
\hline Collaboration, communication, and coordination & $\begin{array}{l}\text { Longo et al. (2019), Saberi et al. (2019a, b), Swan } \\
\text { (2015) }\end{array}$ \\
\hline Cultural differences & Saberi et al. (2019a, b) \\
\hline Customers' awareness & Saberi et al. (2019a, b) \\
\hline Ethical industry involvement & Saberi et al. (2019a, b) \\
\hline External stakeholders' involvement & Kouhizadeh \& Sarkis (2018), Saberi et al. (2019a, b) \\
\hline Financial constraints & Iansiti \& Lakhani, (2017), Wang et al. (2019) \\
\hline Governmental policies & Saberi et al. (2019a, b), Swan (2015) \\
\hline High sustainability costs & Biswas \& Gupta (2019), Yli-Huumo et al. (2016) \\
\hline Immaturity & Saberi et al. (2019a, b) \\
\hline Immutability & Asadi Bagloee et al. (2019), Ghode et al. (2020) \\
\hline Implementation tools & Saberi et al. (2019a, b), Swan (2015) \\
\hline Information disclosure policy & Choi et al. (2020), Saberi et al. (2019a, b) \\
\hline Knowledge and expertise & Helo \& Hao (2019), Saberi et al. (2019a, b) \\
\hline Legal and regulatory uncertainties & Beck et al. (2018), Biswas \& Gupta (2019) \\
\hline Managerial commitment & Saberi et al. $(2019 a, b)$ \\
\hline Market competition and demand uncertainty & Saberi et al. (2019a, b) \\
\hline Negative public perception & Saberi et al. $(2019 a, b)$ \\
\hline Organizational culture change & Saberi et al. (2019a, b), Swan (2015) \\
\hline Organizational policies & Ghode et al. (2020), Saberi et al. (2019a, b) \\
\hline Poor economic behavior in the long run & Biswas \& Gupta (2019) \\
\hline Privacy risks & $\begin{array}{l}\text { Biswas \& Gupta (2019), Swan (2015), Wang et al. } \\
\text { (2019) }\end{array}$ \\
\hline Rewards and encouragement & Beck et al. (2018), Saberi et al. (2019a, b) \\
\hline Risks of cyber-attacks & Biswas \& Gupta (2019), Ghode et al. (2020) \\
\hline Security & Biswas \& Gupta (2019), Saberi et al. (2019a, b) \\
\hline Sustainable practices integration & Kouhizadeh \& Sarkis (2018), Saberi et al. (2019a, b) \\
\hline System conversion hesitation & Saberi et al. (2019a, b) \\
\hline
\end{tabular}

The elements in the pairwise comparison matrices are replaced with the corresponding SVTNNs in accordance with the scale shown in Table 3 (see Definition 7 in "Appendix" to calculate the inverse of a SVTNN).

Step 5. Aggregating opinions of the experts in the SVTNNs:

The trapezoidal neutrosophic weighted arithmetic averaging (TNWAA) operator is used to aggregate the opinions of the various experts to determine their weighting, as described in Definition 8 in "Appendix". The weights are determined based on each expert's knowledge and expertise in the related field.

Step 6. Calculating the neutrosophic synthetic values:

The neutrosophic synthetic value of each element, which is shown by $S_{i}$, is calculated using Eq. (3). 
Fig. 2 Steps in the research methodology
Research steps

1

Literature review

2

Initial list of barriers

3

Expert panel

4

Final list of barriers

5

N-AHP application

6

Final order of barriers

$$
S_{i}=\sum_{j=1}^{n} \eta_{i j} \times\left[\sum_{i=1}^{n} \sum_{j=1}^{n} \eta_{i j}\right]^{-1} i=1, \ldots, n
$$

Here, $n$ is the number of elements, and $\eta_{i j}$ is the $(i, j)^{t h}$ element of the aggregated pairwise comparison matrix, which is in SVTNNs.

Step 7. Determining the final importance-weights:

The final importance-weights are calculated with Eq. (4) and are indicated by $W_{i}$ in SVTNNs. To compare the weights, they should be converted to crisp values based on the score function as defined in Definition 9 in "Appendix".

$$
W_{i}=\frac{S_{i}}{\sum_{i=1}^{n} S_{i}} i=1, \ldots, n
$$


Table 3 The proposed neutrosophic rating scale in N-AHP

\begin{tabular}{llll}
\hline $\begin{array}{l}\text { Numerical } \\
\text { scale }\end{array}$ & Verbal scale & SVTNNs & Score function \\
\hline$\frac{1}{9}$ & Extremely less important & $<(0.11,0.11,0.11,0.11) ; 1,0,0>0.11$ \\
$\frac{1}{8}$ & Very very strongly less important & $<(0.11,0.11,0.13,0.14) ; 1,0,0>0.12$ \\
$\frac{1}{7}$ & Very strongly plus less important & $<(0.11,0.13,0.14,0.17) ; 1,0,0>$ & 0.14 \\
$\frac{1}{6}$ & Strongly plus less important & $<(0.13,0.14,0.17,0.2) ; 1,0,0>$ & 0.16 \\
$\frac{1}{5}$ & Strongly less important & $<(0.14,0.17,0.2,0.25) ; 1,0,0>$ & 0.19 \\
$\frac{1}{4}$ & Moderately plus less important & $<(0.17,0.20,0.25,0.33) ; 1,0,0>$ & 0.24 \\
$\frac{1}{3}$ & Moderately less important & $<(0.14,0.17,0.33,0.50) ; 1,0,0>$ & 0.29 \\
$\frac{1}{2}$ & Weakly less important & $<(0.20,0.25,0.5,1) ; 1,0,0>$ & 0.49 \\
1 & Equally important & $<(1,1,1,1) ; 0.5,0.5,0.5>$ & 0.5 \\
2 & Weakly more important & $<(1,2,4,5) ; 0.4,0.65,0.6>$ & 1.15 \\
3 & Moderately more important & $<(2,3,6,7) ; 0.3,0.75,0.7>$ & 1.28 \\
4 & Moderately plus more important & $<(3,4,5,6) ; 0.6,0.35,0.4>$ & 2.78 \\
5 & Strongly more important & $<(4,5,6,7) ; 0.8,0.15,0.2>$ & 4.49 \\
6 & Strongly plus more important & $<(5,6,7,8) ; 0.7,0.25,0.3>$ & 4.66 \\
7 & Very strongly plus more important & $<(6,7,8,9) ; 0.9,0.1,0.1>$ & 6.75 \\
8 & Very very strongly more important & $<(7,8,9,9) ; 0.85,0.1,0.15>$ & 7.15 \\
9 & Extremely more important & $<(9,9,9,9) ; 1,0,0>$ & 9 \\
\hline & & &
\end{tabular}

\section{Real-world application and analysis}

Six different cases from Iranian manufacturing companies were selected for this study. Manufacturing accounts for most of Iran's GDP after oil and gas (Badri Ahmadi et al., 2017). The six case companies were automotive, chemical, cement, tile, telecom, and electronics manufacturing firms. Experts who were invited to participate in this study either held upper-level management positions or were trained SC professionals operating under the supervision of highly qualified international consultants. They were knowledgeable professionals in their field with extensive work experience. We compiled a shortlist of experts with the most relevant knowledge regarding blockchain technology and SCs from different functional areas. We carried out informative discussions with several corporations and invited a panel of experts to participate in the study. The team members informed the experts about the research goals. The experts involved in this study gained knowledge and understanding of the topic and expressed interest in assessing blockchain technology implementation in their SCs. A description of the case companies and assigned managers is given in Table 4.

\subsection{Development of the evaluation framework}

The evaluation framework in the study was developed based on a comprehensive literature review where potential blockchain barriers were identified (Table 2). Subsequently, a survey was designed and sent to experts for their review. They were asked to vote for each 
Table 4 Experts involved in blockchain technology barrier analysis and their importance-weights

\begin{tabular}{llll}
\hline Expert no. and Industry & Position & $\begin{array}{l}\text { Experience } \\
\text { (Years) }\end{array}$ & $\begin{array}{l}\text { Importance } \\
\text { Weights }\end{array}$ \\
\hline Expert 1 (Automotive manufacturing) & Supply manager & 14 & 0.10 \\
Expert 2 (Chemical manufacturing) & Purchasing manager & 11 & 0.05 \\
Expert 3 (Cement manufacturing) & Logistics manager & 25 & 0.30 \\
Expert 4 (Tile manufacturing) & IT manager & 18 & 0.20 \\
Expert 5 (Telecom manufacturing) & Production manager & 14 & 0.10 \\
Expert 6 (Electronics manufacturing) & Financial manager & 20 & 0.25 \\
\hline
\end{tabular}

barrier and determine which of the barriers were relevant to their SCs by indicating Yes (as accepted or approved) or No (as rejected or not approved). The authors agreed with the experts that those barriers that were approved by at least 5 experts would be considered for the next round of review. In total, three rounds of reviews were conducted to refine the set of barriers. Experts were also asked to add any additional barriers that were relevant to their SC based on their knowledge and expertise. Two additional barriers, namely, transaction-level uncertainties and usage in the underground economy, were suggested by two of the experts. Eventually, five barriers were selected and included in the final list, as shown in Table 5.

\subsection{N-AHP application}

The N-AHP is applied to obtain the weights of the five barriers: B1 (transaction-level uncertainties), B2 (usage in the underground economy), B3 (challenges in scalability), B4 (privacy risks), and B5 (managerial commitment). Thus, the decision hierarchy in this current problem is comprised of one level. Table 6 shows the initial pairwise comparison matrices based on the data obtained from the six experts using the neutrosophic rating scale (Table 3$)$ provided $\left(A_{1}, \ldots, A_{6}\right)$.

Table 5 Final list of barriers to blockchain technology

\begin{tabular}{ll}
\hline Barriers to blockchain technology & Description \\
\hline Transaction-level uncertainties (B1) & $\begin{array}{c}\text { Refers to the uncertainties in blockchain application at the } \\
\text { micro-level. For example, how many transactions can be } \\
\text { recorded in a blockchain and what information can be shared } \\
\text { to preserve a certain level of privacy }\end{array}$ \\
Usage in the underground economy (B2) & $\begin{array}{l}\text { Refers to the employment of blockchain as a payment method in } \\
\text { money laundering activities such as gambling, which are high } \\
\text { risk, as these blockchain transactions cannot be controlled }\end{array}$ \\
Rhallenges in scalability (B3) & $\begin{array}{l}\text { Refers to technical barriers and means that each block is not } \\
\text { capable of processing millions of transactions in a real-time } \\
\text { setting and the difficulty with consensus protocols }\end{array}$ \\
Privacy risks (B4) & $\begin{array}{l}\text { Refers to privacy and security issues in blockchain networks and } \\
\text { transactions }\end{array}$ \\
Ranagerial commitment (B5) & concerning implementation and in dealing with the potential \\
& risks of blockchain technology
\end{tabular}


Table 6 The initial pairwise comparison matrices for the six experts

\begin{tabular}{lllllllllllll}
\hline & & B1 & B2 & B3 & B4 & B5 & & B1 & B2 & B3 & B4 & B5 \\
\hline $\boldsymbol{A}_{1}$ & B1 & 1 & 1 & 2 & 3 & $1 / 3$ & $A_{2}$ & 1 & 1 & 2 & 2 & $1 / 3$ \\
& B2 & 1 & 1 & 3 & 2 & 2 & & 1 & 1 & 3 & 4 & 2 \\
& B3 & $1 / 2$ & $1 / 3$ & 1 & 1 & $1 / 2$ & & $1 / 2$ & $1 / 3$ & 1 & 1 & $1 / 2$ \\
& B4 & $1 / 3$ & $1 / 2$ & 1 & 1 & $1 / 5$ & & $1 / 2$ & $1 / 4$ & 1 & 1 & 1 \\
& B5 & 3 & $1 / 2$ & 2 & 5 & 1 & & 3 & $1 / 2$ & 2 & 1 & 1 \\
$\boldsymbol{A}_{3}$ & B1 & 1 & 1 & 2 & 5 & 1 & $A_{4}$ & 1 & 5 & 2 & 5 & 3 \\
& B2 & 1 & 1 & 3 & 2 & 2 & & $1 / 5$ & 1 & 1 & 2 & 2 \\
& B3 & $1 / 2$ & $1 / 3$ & 1 & 1 & $1 / 2$ & & $1 / 2$ & 1 & 1 & 1 & 2 \\
& B4 & $1 / 5$ & $1 / 2$ & 1 & 1 & 1 & & $1 / 5$ & $1 / 2$ & 1 & 1 & 2 \\
& B5 & 1 & $1 / 2$ & 2 & 1 & 1 & & $1 / 3$ & $1 / 2$ & $1 / 2$ & $1 / 2$ & 1 \\
$\boldsymbol{A}_{5}$ & B1 & 1 & 1 & 2 & 1 & 1 & $A_{6}$ & 1 & 3 & 2 & 1 & 2 \\
& B2 & 1 & 1 & 3 & 2 & 2 & & $1 / 3$ & 1 & 1 & 2 & 2 \\
& B3 & $1 / 2$ & $1 / 3$ & 1 & 1 & 2 & & $1 / 2$ & 1 & 1 & 1 & 3 \\
& B4 & 1 & $1 / 2$ & 1 & 1 & 1 & & 1 & $1 / 2$ & 1 & 1 & 2 \\
& B5 & 1 & $1 / 2$ & $1 / 2$ & 1 & 1 & & $1 / 2$ & $1 / 2$ & $1 / 3$ & $1 / 2$ & 1 \\
\hline
\end{tabular}

The CRs calculated by Eq. (2) for each pairwise comparison matrix are $9.31 \%, 8.96 \%$, $5.47 \%, 7.66 \%, 5.26 \%$, and $7.37 \%$. The CR values are all below $10 \%$, indicating consistent evaluations. Next, the values of the initial pairwise comparison matrices are replaced with the corresponding SVTNNs based on Table 3. The aggregation neutrosophic matrix is calculated by TNWAA as explained in Sect. 3, step 5 and "Appendix", Definition 8. Then, the final weights and ranking are obtained by applying Eqs. (3) and (4), as shown in Table 7.

\section{Results}

\subsection{Transaction-level uncertainties (B1)}

The first main observation from our findings is that transaction-level uncertainties are the most critical barriers for developing blockchain technology in Iranian manufacturing SCs because they have the highest weight (i.e., 0.321). Biswas \& Gupta (2019) highlighted the importance of this barrier in blockchain deployment and noted that transaction-level uncertainties happen because of mistaken transactions, the possibility of the cancellation of confirmed transactions, or having no option but to blacklist suspicious DLT blocks. Jabbar

Table 7 Final weights and ranking of barriers obtained from N-AHP

\begin{tabular}{llll}
\hline Barriers to blockchain technology & SVTNN weights & $\begin{array}{l}\text { Normalized } \\
\text { crisp weights }\end{array}$ & Ranking \\
\hline Transaction-level uncertainties (B1) & $<(0.06,0.14,0.75,1.78) ; 1,0,0>$ & 0.321 & 1 \\
Usage in the underground economy (B2) & $<(0.04,0.11,0.67,1.62) ; 1,0,0>$ & 0.287 & 2 \\
Challenges in scalability (B3) & $<(0.03,0.06,0.30,0.75) ; 1,0,0>$ & 0.134 & 4 \\
Privacy risks (B4) & $<(0.03,0.06,0.26,0.63) ; 1,0,0>$ & 0.115 & 5 \\
Managerial commitment (B5) & $<(0.03,0.06,0.31,0.82) ; 1,0,0>$ & 0.143 & 3 \\
\hline
\end{tabular}


\& Dani (2020) studied the link between blockchain transactions and computational costs. According to Möser et al. (2013), users of blockchains involved in illegal activities might be blacklisted by law agencies in the future. Thus, transaction-level uncertainties (B1) can be a major obstacle for the successful implementation of blockchain, as has been confirmed in the literature (Cohen \& Zohar, 2018). Transaction-level uncertainties highlight the fact that managers in the case companies, as well as other Iranian manufacturing firms, should become more aware of the significant benefits their firms can gain through developing appropriate strategies to effectively mitigate this barrier.

\subsection{Usage in the underground economy (B2)}

This barrier is second in importance, with a weight of 0.287. Managers in the Iranian manufacturing sector need to recognize the high risk and threat of using blockchain networks as a payment method for underground activities, such as gambling and money laundering, since these activities are too complex to be monitored and controlled by authorities. Although blockchain is generally a secure technology, occasional security gaps can render it susceptible to hacking (Iansiti \& Lakhani, 2017). According to Vukolić (2016), there is considerable price inconsistency in the blockchain market. Hastig and Sodhi (2020) identified the curbing of illegal practices as one of the business requirements for traceability systems in supply chains. Nefarious usage of bitcoin, which is associated with blockchain technology, has been highlighted as a problem in the literature (Wang et al., 2019). This result is supported by several studies showing that usage in the underground economy is a major barrier to the deployment of blockchain technology (Vukolić, 2016).

\subsection{Managerial commitment (B5)}

This barrier is third in the final ranking list with a weight of 0.143 . In the international supply chain management (ISCM) context, Akkermans et al. (1999) identified a lack of managerial attention and prevailing dominance of functional thinking as barriers to effective SCM. They also discussed that functional thinking barriers are likely to be positively correlated with the geographical dispersion of facilities. Akkermans et al. (1999) pointed out that synchronization of processes in SCs can never rely solely on technological progress. Proper organizational change programs are also necessary. Thus, dealing with managerial commitment in a developing economy has its own specifications, which are different from other managerial contexts and cultures. The implications of our findings might be partly related to Hofstede's cultural dimensions. For instance, uncertainty avoidance is the highest of Hofstede's cultural dimensions in Iran (Moghadam \& Assar, 2008). The dominant cultural preference is to avoid uncertainty. In other words, the culture is intolerant to unorthodox ideas, which might be linked to the implementation of new technologies such as blockchains and the uncertainties arising from their usage in the underground economy.

\subsection{Challenges in scalability (B3)}

This is ranked fourth (weight 0.134). Prior studies highlighted scalability issues of blockchain technology, such as data storage, communication malfunctions, and linear transaction records (Esmaeilian et al., 2020). Biswas \& Gupta (2019) identified the most influential barriers as challenges in scalability and market-based risks. They indicated that blockchain 
networks see considerable challenges in scalability that contain slow transaction processing and block-size limitations. Vatankhah Barenji et al. (2020) indicated the issue of scalability problems in small and medium manufacturing enterprises (SMEs).

\subsection{Privacy risks (B4)}

This risk is ranked last with a weight of 0.115 based on the final results. One common blockchain myth is that it is a $100 \%$ secure technology. In reality, blockchain's security depends on the breaching of adjacent applications (Carson et al., 2018). The potential security and privacy risks of blockchain technology have been discussed in the literature (Zhang et al., 2019).

\section{Discussion}

\subsection{Theoretical contributions}

Decision support tools such as MCDA methods are invaluable business analytics methods helping organizations make decisions in complex operating environments. Among the MCDA methods, AHP is one of the most commonly practiced, mainly because of its ease of application and flexibility for integration with various other methods. The inclusion of subjective factors has been considered one of the AHP's advantages over other MCDA methods (Emrouznejad \& Marra, 2017). Many studies have focused on the fuzzy set-based extension of the AHP, namely, fuzzy AHP (F-AHP), to capture uncertainty (Emrouznejad $\&$ Ho, 2017; Ecer, 2020; Sitorus et al., 2019). However, few articles have investigated the extension of the AHP to other uncertainty theories, such as NST, which can enhance its capability for formulating a better decision-making process under uncertainty. There have been few recent developments and applications of AHP and NST that benefit from their own specific characteristics (Abdel-Basset et al., 2018; Bolturk \& Kahraman, 2018).

This paper contributes to the MCDA literature in several aspects. Here, we explain the strengths of the proposed method in comparison to other similar extensions of the AHP. First, an extension of the AHP method in the uncertain environment of NST (i.e., N-AHP) is proposed in this study, which utilizes SVTNNs for the first time, necessitating the introduction of mathematical subtraction, division, and inverse SVTNN operators (see "Appendix"). In previous studies, triangular neutrosophic numbers (TNNs) were integrated with the AHP, which is different from SVTNNs (Abdel-Basset et al., 2018). Bolturk \& Kahraman (2018) proposed a novel interval-valued neutrosophic AHP (IVN-AHP) based on cosine similarity measures. Abdel-Basset et al. (2018) combined the AHP and the Delphi method in a neutrosophic environment to check consistency and compute the degree of consensus among experts. We found no study dealing with the integration of SVTNNs and AHP in the literature, which makes the proposed method a unique approach in a group decision-making context. Second, the NST can independently quantify the indeterminacy membership and, unlike fuzzy set theory, can present information about rejection (Vafadarnikjoo et al., 2020). The NST has improved the intuitionistic fuzzy set (IFS), which was initially proposed by Atanassov (1986). Smarandache (2005) thoroughly elaborated on the distinctions between NST and IFS and emphasized advantages such as the ability to quantify the indeterminacy membership function, independent of membership and nonmembership functions. This advantage, along with the ability to express the information about 
rejection that was not possible with integration with fuzzy set theory, would allow analysts to capture uncertainty in the subjective judgments of experts more accurately in an NST decision-making environment.

\subsection{Theoretical implications}

The proposed N-AHP method is a unique and reliable methodology that can be used to capture experts' subjective uncertainty in relation to the analysis of blockchain adoption barriers in manufacturing supply chain settings in the context of a developing economy. As expert knowledge is based on subjective judgments, especially in a novel field such as blockchain technology, the application of the hybrid N-AHP method can bring significant benefits in terms of theoretical implications (see contributions of the proposed N-AHP in Sect. 6.1.).

Apart from the methodological advantages of the 2-step approach for the analysis of blockchain success introduced by the Boston consulting group (BCG) (Bender et al., 2019), we believe our findings show the benefits of using the blockchain validation step to help companies in the manufacturing SC successfully implement blockchain technology. In other words, companies can assess and implement the appropriate blockchain technology for their businesses more efficiently by evaluating the most critical implementation barriers. The theoretical implications of our work (analyzing blockchain barriers) contribute to both blockchain technological and commercial validation, as shown in Fig. 3. Conceptually, we adopted Bender et al. (2019) model and extended it by including a blockchain adoption
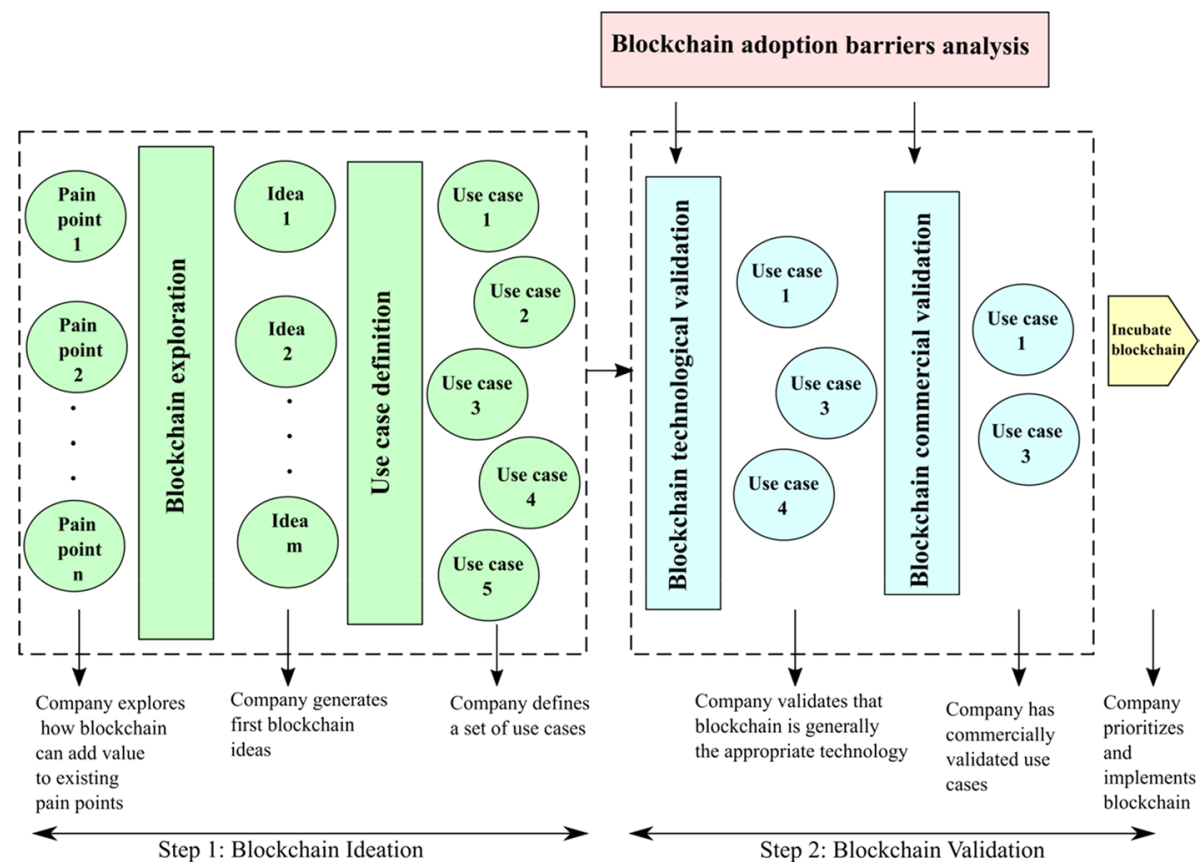

Fig. 3 Two-step approach to successful blockchain implementation [ Adapted from BCG in Bender et al. (2019)] 
barrier analysis. The base model by Bender et al. (2019) proposed a two-step approach for companies to overcome the complexities of blockchain implementation. Based on our results, we believe that the suggested extension, blockchain adoption barriers analysis, is a useful expansion to the original model (Bender et al., 2019). Based on this theoretical model and the obtained findings, a proposed action plan framework for blockchain validation was developed and is explained in the practical implications section (Sect. 6.3.).

In step 1, companies are required to seek out current existing pain points within their supply chains. To obtain the greatest payback from blockchain, companies should target the most critical pain points. Then, they should develop ideas through which blockchain capabilities could generate meaningful value before defining a set of blockchain use cases. After obtaining a set of possible use cases, companies need to evaluate whether blockchain is the best choice for attaining their organizational objectives, whether there might be another better alternative solution, and whether proceeding with that technology would be commercially interesting. As part of the validation step, companies can refer to our findings to understand what major barriers they might encounter during blockchain implementation. Finally, after the completion of these two steps, the validated blockchain use cases are ready for incubation (Bender et al., 2019).

\subsection{Practical implications}

As discussed in the results section, transaction-level uncertainties, usage in the underground economy, managerial commitment, challenges in scalability, and privacy risks are identified as the main barriers within our study. The findings are consistent with the scant literature on blockchain barriers in a developing economy. Based on the theoretical implications and using the two-step approach (Fig. 3), our findings contribute to the validation of the successful implementation of blockchains in a developing economy. To provide action plans based on the identified barriers, we need to focus first on the chosen growth model in businesses and then on each of the prioritized barriers and provide strategies to pave the way toward blockchain validation success (Fig. 4). Prior research has warned against the inappropriate and unnecessary use of blockchain technology, which might cause serious internal and external disruptions in addition to incurring considerable costs (Angelis \& Ribeiro da Silva, 2019). It is suggested that firms in developing economies follow the twostep approach (Fig. 3) to confirm how and why blockchain is a suitable solution for their organization, as shown in the ideation step. Thus, our findings can help them overcome potential barriers.

Select the proper value creation growth model: Chong et al. (2019) discussed five business models to capture the value of blockchain. Bender et al. (2019) discussed two primary growth models for value creation by implementing blockchain solutions: (1) linear (incremental win) and (2) network effects (true disruptor). In the linear growth model, the absolute value is limited to a company's production or sales capacities, and users derive value instantly after using the product (such as pens for writing). On the other hand, with network effects, growth is unbundled from production and sales. A good example of this is the telephone, where the overall value for the first users (of the telephone) is limited because people can only call other telephone owners (Bender et al., 2019). Companies applying blockchain application cases that deliver network effects have the potential to be true disruptors (Bender et al., 2019). These true-disruptor application cases require significant financial and technical resources, the ability to deal with 


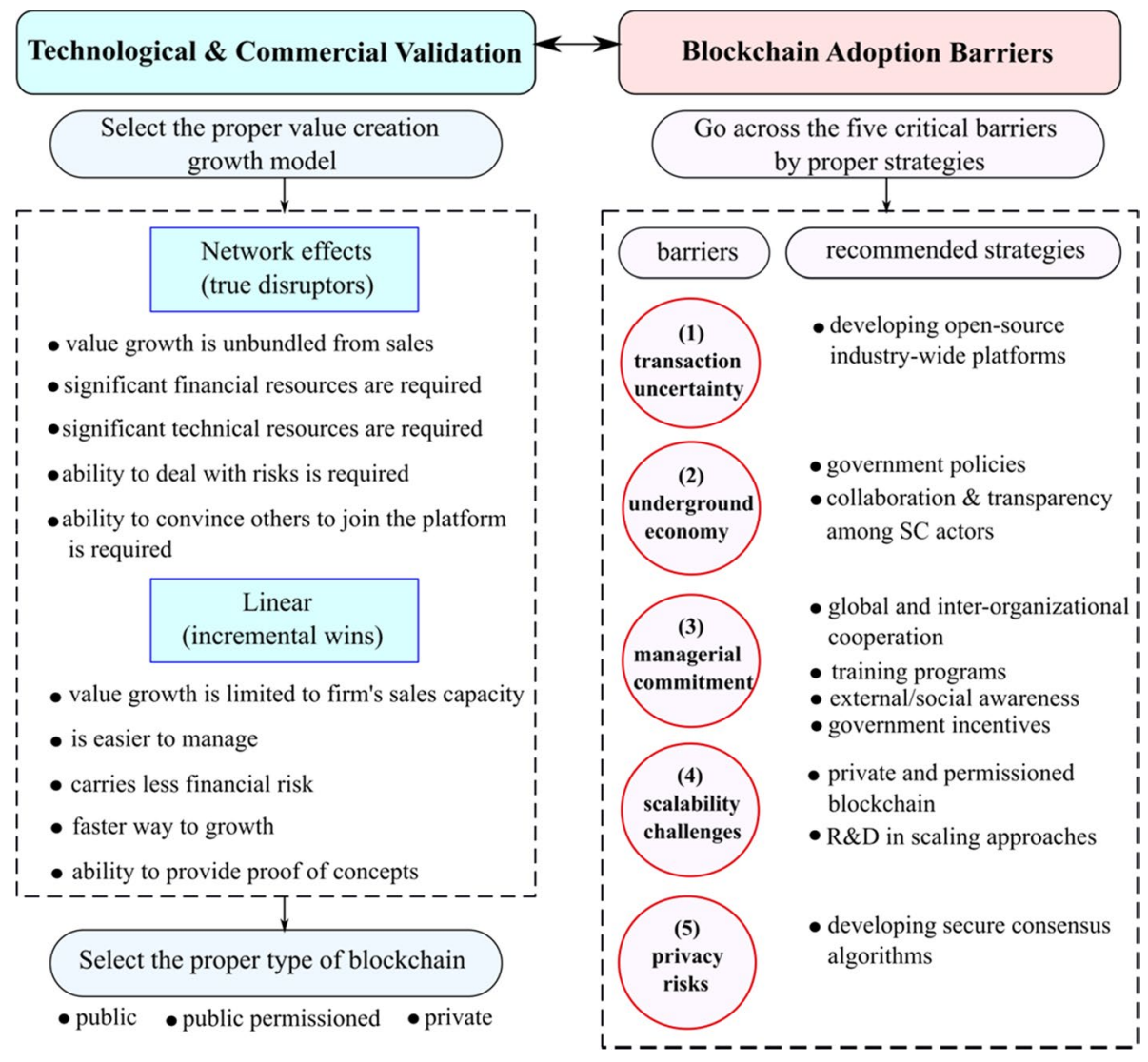

Fig. 4 Action plan framework for blockchain validation in a developing economy

risks, and the ability to convince others to join the platform. This model requires participants to accept new standards that usually require managerial involvement in supply chains and a strong regulatory push, which all require serious effort in a developing economy like Iran's. However, pursuing incremental-win application cases based on a linear model, where each incremental participant or transaction linearly adds value, can be a suitable alternative in developing economies in the short-term. The reason is that incremental wins concentrate on a linear growth model that is easier to manage, especially considering that one of the critical identified barriers in our study was managerial commitment. Second, incremental wins carry lower financial risk and can offer a faster way to grow. Third, incremental wins can provide proof of concept to attract the attention of partners and validate their participation in a blockchain-enabled solution (Bender et al., 2019).

Select the proper type of blockchain: Ganeriwalla et al. (2018) pointed out that blockchain technology can be categorized as public, public-permissioned, or private. Bitcoin is an example of a public blockchain that is open, and anyone with computing capacity can contribute to the network, keep the ledger, and weigh in on consensus issues. Conversely, private and public-permissioned types of blockchain platforms are controlled by 
a handful of businesses with the ultimate say in all aspects of the platform. Although the majority of industries are headed towards private and public-permissioned implementation, this trend has garnered some criticism, and companies in developing economies should take all the pros and cons into consideration (Esmaeilian et al., 2020).

Using the proper strategies to overcome the five critical barriers: (1) Transaction uncertainty: SmartLog is a recent proof of concept platform for blockchain applications in logistics and supply chains that enables various partners within the industry to obtain access to real-time logistics information (Esmaeilian et al., 2020). The implementation of similar open-source industry-wide platforms would reduce transaction-level uncertainties in logistics, particularly in developing economies where a lack of visibility in logistics and supply chain operations is the norm. Kane (2017) argued that although blockchain is an emerging technology, it has many of the key features of a general-purpose technology (GPT), including the capability for further improvement, a pervasiveness in various sectors of the economy, and the ability to facilitate the creation of new innovations. However, logistics operators in practice, particularly those in small and medium enterprises (SMEs), have limited knowledge of blockchains (Petersen et al., 2018). This was also noted in our study. The reason for this lack of familiarity by SMEs might be due to the speed of blockchain adoption. Blockchain is a foundational technology that has been adopted gradually over a period of time; it may require years to change the SC landscape (Iansiti \& Lakhani, 2017). (2) Underground economy: Luthra et al. (2020) noted that government encouragement policies, collaboration, and transparency among SC actors are extremely important enablers of Industry 4.0 from an emerging economy perspective. (3) Managerial commitment: As discussed in the results section, soliciting the total support of management is imperative for blockchain implementation. This can be achieved by training and proper organizational change programs, global and interorganizational cooperation, government incentives, and external/social awareness. (4) Scalability challenges: As the number of users and the size of blockchain platforms grow, the need to deal with scalability issues becomes more apparent (Esmaeilian et al., 2020). Various scaling approaches have been developed in computer science to overcome scalability issues. Scalable networks have the capability to transfer information between intermediaries with no need to record all transactions on the blockchain (Xie et al., 2019). Thus, more R\&D investment in advancing scaling approaches can help in crossing this barrier. Moreover, Carson et al. (2018) pointed out that private and permissioned blockchains have the capability to optimize network openness and scalability. (5) Privacy risks: Zhang et al. (2019) recommended that more secure consensus algorithms be required to improve the security and privacy of blockchain systems.

Companies and investors are dealing with the issue of how blockchain can be molded to create value. Bender et al. (2019) looked "at the example of the internet" and compared it to blockchain technology. They found that just as it took a while for organizations to realize how to turn the power of the internet into growth and value, the same is happening for blockchain technology. Iansiti and Lakhani (2017) pointed out that blockchain technology is decades from reaching its full potential. This issue of development can be much more troublesome in developing economies, triggering lower managerial commitment because managers are often too focused on profitability and might take a conservative view toward new technologies such as blockchain.

Managers need to ensure meaningful returns from their investments. Carson et al. (2018) analyzed and quantified monetary impact in more than 90 use cases (14 industries) in terms of four value indices: cost reduction, revenue generation, capital relief, and social 
value. They found that nearly $70 \%$ of the value at stake in the short run across industries, including manufacturing, is primarily in cost reduction. This point can be particularly important to take into consideration for companies in manufacturing SCs; however, more investigation is needed to fully confirm that the same trend exists in the context of developing economies.

\subsection{Limitations}

This research suffers from some limitations. First, this study employed N-AHP, which required recruiting experts for primary data collection from case companies, which might hinder the generalizability of the results. However, the impact of this limitation can be minimized with additional studies or by extending the number of experts or case companies to provide confirmation or rebuttal of the findings in the future. The number of experts involved in the current study and the determination of the experts' importance-weights can be defined and justified more systematically by introducing an expert selection model. The second limitation is related to the fact that blockchain technology is in its infancy, both in terms of research and practice. Exploring blockchain applicability in a developing economy is even more difficult because few organizations are willing to spend time and money on this disruptive technology. This made it difficult to find experts who could properly evaluate the barriers. The third limitation is related to the practical applications of the findings. Specifically, there must be a substantial shift in the entrenched mindsets and practices of managers and policymakers from classical information management systems in traditional manufacturing SCs to the integration of blockchain technology, which necessitates organizational change programs. Blockchain technology takes more time for adoption and requires considerable perseverance and exertion.

\section{Conclusions}

This study has advanced knowledge about barriers to blockchain technology implementation in the SC literature in an emerging economy context (i.e., Iran). An action plan framework for blockchain validation in a developing economy was also proposed to provide practical insights. Initially, several potential barriers to blockchain technology were identified based on the literature and were subjected to review by industrial experts, with the target of constructing a decision framework. A sample of six Iranian manufacturing experts was employed in the assessment and decision-making process. The framework included five barriers: transaction-level uncertainties (B1), usage in the underground economy (B2), challenges in scalability (B3), privacy risks (B4), and managerial commitment (B5). This work proposed and applied a method, namely, N-AHP, to assess and rank the most relevant barriers to blockchain technology according to their importance-weight in the context of Iran's six manufacturing industries (i.e., automotive, chemical, cement, tile, telecom, and electronics manufacturing). The findings revealed that transaction-level uncertainties (B1), usage in the underground economy (B2), managerial commitment (B5), challenges in scalability (B3), and privacy risks (B4) are the most critical barriers, in order of importance. Managers and policymakers in the examined case companies can make significant practical contributions to manufacturing SCs for blockchain technology implementation by considering the proposed action plan framework. From a practical perspective, our study enables 
scholars and practitioners to further their understanding of the most critical barriers they will face when attempting to implement blockchain technology in a developing economy..

Given the merits of blockchain technology, businesses should be aware of the fact that first, there will be a dramatic change in how businesses will function in the next few decades, and sooner or later, developing economies should also follow this trend. Second, similar to prior technological shifts, early movers will reap the greatest benefits from it by expanding partnerships, setting standards, and advancing technology adoption. However, Jeffers (2010) argued that merely owning a rare and valuable IT resource that competitors cannot easily replicate does not necessarily guarantee a competitive advantage. He indicated that the wider concerns of the environmental and social aspects of sustainability must also be embraced in the strategy paradigm. This opens up a new future research avenue where sustainability concerns are taken into consideration for blockchain technology implementation. Future works can also investigate the interrelationships between identified barriers to understand which barriers act as causes and which barriers are affected by other factors by applying methods such as DEMATEL. In future studies, triangulation by applying similar methods to the AHP, such as the best-worst method (BWM), can increase the validity of the findings. In addition, more empirical studies are necessary to confirm the suggested practical strategies in our proposed action plan framework to effectively deal with the identified barriers within the context of a developing economy. Replicating the research in other geographical regions can contribute to international comparisons, as many barriers to effective ISCM are deeply embedded in the organizational structures and cultures of firms, and no process synchronization is possible across SCs by merely relying on technological advancements. More studies on organizational practices around the adoption of blockchain technology are needed, as this area of research is in its early phases, particularly in emerging economies.

\section{Appendix: Neutrosophic set theory (NST)}

Some basic definitions of NST are provided in this section to aid in understanding the implementation of N-AHP.

Definition 1 Neutrosophic set (NS) (Vafadarnikjoo, 2020). Let $U$ be a finite set of objects, and let $x$ signify a generic element in $U$. The NS $A$ in $U$ is characterized by a truthmembership function $T_{A}(x)$, an indeterminacy-membership function $I_{A}(x)$, and a falsitymembership function $F_{A}(x) . T_{A}(x), I_{A}(x)$, and $F_{A}(x)$ are the elements of $] 0^{-}, 1^{+}[$. It can be shown as Eq. (5):

$$
A=\left\{\left\langle x,\left(T_{A}(x), I_{A}(x), F_{A}(x)\right)\right\rangle: x \in U, T_{A}(x), I_{A}(x), F_{A}(x) \in\right] 0^{-}, 1^{+}[\}
$$

Note that $0^{-} \leq T_{A}(x)+I_{A}(x)+F_{A}(x) \leq 3^{+}$.

Definition 2 Single-valued neutrosophic set (SVNS) (Vafadarnikjoo, 2020). Let $U$ be a finite set of elements, and let $x$ signify a generic element in $U$. An SVNS $A$ in $U$ is defined by a truth-membership function $T_{A}(x)$, an indeterminacy-membership function $I_{A}(x)$, and a falsity-membership function $F_{A}(x) . T_{A}(x), I_{A}(x)$, and $F_{A}(x)$ are the elements of $[0,1]$. It can be shown as Eq. (6): 


$$
A=\left\{\left\langle x,\left(T_{A}(x), I_{A}(x), F_{A}(x)\right)\right\rangle: x \in U, T_{A}(x), I_{A}(x), F_{A}(x) \in[0,1]\right\}
$$

Note that $0 \leq T_{A}(x)+I_{A}(x)+F_{A}(x) \leq 3$.

For convenience, an SVNS $\left.A=\left\{<x,\left(T_{A}(x), I_{A}(x), F_{A}(x)\right)\right\rangle: x \in U\right\}$ is sometimes shown as a $A=\left\{<T_{A}(x), I_{A}(x), F_{A}(x)>: x \in U\right\}$ in simplified form.

Definition 3 Single-valued trapezoidal neutrosophic number (SVTNN) (Deli \& Subas, 2014). An SVTNN $\tilde{a}=<\left(a_{1}, b_{1}, c_{1}, d_{1}\right) ; w_{\tilde{a}}, u_{\tilde{a}}, y_{\tilde{a}}>, a_{1}, b_{1}, c_{1}, d_{1} \in R, a_{1} \leq b_{1} \leq c_{1} \leq d_{1}$, and $w_{\tilde{a}}, u_{\tilde{a}}, y_{\tilde{a}} \in[0,1]$ is a particular single-valued neutrosophic number (SVNN) whose $T_{\tilde{a}}(x), I_{\tilde{a}}(x)$, and $F_{\tilde{a}}(x)$ are presented as Equations (7) to (9), respectively.

$$
\begin{gathered}
T_{\tilde{a}}(x)=\left\{\begin{array}{c}
\left(x-a_{1}\right) w_{\tilde{a}} /\left(b_{1}-a_{1}\right) a_{1} \leq x<b_{1} \\
w_{\tilde{a}} b_{1} \leq x \leq c_{1} \\
\left(d_{1}-x\right) w_{\tilde{a}} /\left(d_{1}-c_{1}\right) c_{1}<x \leq d_{1} \\
0 \text { otherwise }
\end{array}\right. \\
I_{\tilde{a}}(x)=\left\{\begin{array}{c}
\left(b_{1}-x+u_{\tilde{a}}\left(x-a_{1}\right)\right) /\left(b_{1}-a_{1}\right) a_{1} \leq x<b_{1} \\
u_{\tilde{a}} b_{1} \leq x \leq c_{1} \\
\left(x-c_{1}+u_{\tilde{a}}\left(d_{1}-x\right)\right) /\left(d_{1}-c_{1}\right) c_{1}<x \leq d_{1} \\
1 \text { otherwise }
\end{array}\right. \\
F_{\tilde{a}}(x)=\left\{\begin{array}{c}
\left(b_{1}-x+y_{\tilde{a}}\left(x-a_{1}\right)\right) /\left(b_{1}-a_{1}\right) a_{1} \leq x<b_{1} \\
y_{\tilde{a}} b_{1} \leq x \leq c_{1} \\
\left(x-c_{1}+y_{\tilde{a}}\left(d_{1}-x\right)\right) /\left(d_{1}-c_{1}\right) c_{1}<x \leq d_{1} \\
1 \text { otherwise }
\end{array}\right.
\end{gathered}
$$

Definition 4 Addition of two SVTNNs (Vafadarnikjoo, 2020). Given $\tilde{a}=<\left(a_{1}, b_{1}, c_{1}, d_{1}\right)$; $w_{\tilde{a}}, u_{\tilde{a}}, y_{\tilde{a}}>\quad$ and $\quad \tilde{b}=<\left(a_{2}, b_{2}, c_{2}, d_{2}\right) ; w_{\tilde{b}}, u_{\tilde{b}}, y_{\tilde{b}}>, \quad w_{\tilde{a}}, u_{\tilde{a}}, y_{\tilde{a}}, w_{\tilde{b}}, u_{\tilde{b}}, y_{\tilde{b}} \in[0,1]$, $a_{1}, b_{1}, c_{1}, d_{1}, a_{2}, b_{2}, c_{2}, d_{2} \in \mathbb{R}, a_{1} \leq b_{1} \leq c_{1} \leq d_{1}$, and $a_{2} \leq b_{2} \leq c_{2} \leq d_{2}$, Eq. (10) is true.

$$
\tilde{a}+\tilde{b}=\left\langle\left(a_{1}+a_{2}, b_{1}+b_{2}, c_{1}+c_{2}, d_{1}+d_{2}\right) ; w_{\tilde{a}}+w_{\tilde{b}}-w_{\tilde{a}} w_{\tilde{b}}, u_{\tilde{a}} u_{\tilde{b}}, y_{\tilde{a}} y_{\tilde{b}}\right\rangle
$$

Definition 5 Subtraction of two SVTNNs (Smarandache, 2016). Let $\tilde{a}=<\left(a_{1}, b_{1}, c_{1}, d_{1}\right)$; $w_{\tilde{a}}, u_{\tilde{a}}, y_{\tilde{a}}>$ and $\tilde{b}=<\left(a_{2}, b_{2}, c_{2}, d_{2}\right) ; w_{\tilde{b}}, u_{\tilde{b}}, y_{\tilde{b}}>$ be two SVTNNs and $w_{\tilde{a}}, u_{\tilde{a}}, y_{\tilde{a}}, w_{\tilde{b}}, u_{\tilde{b}}, y_{\tilde{b}} \in[0,1]$ with the restrictions that $w_{\tilde{b}} \neq 1, u_{\tilde{b}} \neq 0, y_{\tilde{b}} \neq 0$, and $a_{1}, b_{1}, c_{1}, d_{1}, a_{2}, b_{2}, c_{2}, d_{2} \in \mathbb{R}, a_{1} \leq b_{1} \leq c_{1} \leq d_{1}$, and $a_{2} \leq b_{2} \leq c_{2} \leq d_{2}$; then, the subtraction of the two SVTNNs is shown in Eq. (11):

$$
\tilde{a}-\tilde{b}=\left\langle\left(a_{1}-d_{2}, b_{1}-c_{2}, c_{1}-b_{2}, d_{1}-a_{2}\right) ; \frac{w_{\tilde{a}}-w_{\tilde{b}}}{1-w_{\tilde{b}}}, \frac{u_{\tilde{a}}}{u_{\tilde{b}}}, \frac{y_{\tilde{a}}}{y_{\tilde{b}}}\right\rangle
$$

Remark: If a component result is less than zero, it is replaced with zero; if a component result is greater than one, it is replaced with one.

Definition 6 Division of two SVTNNs (Smarandache, 2016). Let $\tilde{a}=<\left(a_{1}, b_{1}, c_{1}, d_{1}\right)$; $w_{\tilde{a}}, u_{\tilde{a}}, y_{\tilde{a}}>$, and $\tilde{b}=<\left(a_{2}, b_{2}, c_{2}, d_{2}\right) ; w_{\tilde{b}}, u_{\tilde{b}}, y_{\tilde{b}}>$ be two SVTNNs, where $a_{1}, b_{1}, c_{1}, d_{1}, a_{2}, b_{2}, c_{2}, d_{2}>0, \quad a_{1} \leq b_{1} \leq c_{1} \leq d_{1}, \quad a_{2} \leq b_{2} \leq c_{2} \leq d_{2}, \quad$ and 
$w_{\tilde{a}}, u_{\tilde{a}}, y_{\tilde{a}}, w_{\tilde{b}}, u_{\tilde{b}}, y_{\tilde{b}} \in[0,1]$ with the restrictions that $w_{b} \neq 1, u_{\tilde{b}} \neq 0, y_{b} \neq 0$; then, the division of the two SVTNNs is shown in Eq. (12):

$$
\tilde{a} \div \tilde{b}=\left\langle\left(\frac{a_{1}}{d_{2}}, \frac{b_{1}}{c_{2}}, \frac{c_{1}}{b_{2}}, \frac{d_{1}}{a_{2}}\right) ; \frac{w_{\tilde{a}}}{w_{\tilde{b}}}, \frac{u_{\tilde{a}-} u_{\tilde{b}}}{1-u_{\tilde{b}}}, \frac{y_{\tilde{a}}-y_{\tilde{b}}}{1-y_{\tilde{b}}}\right\rangle
$$

Remark: If a component result is less than zero, it is replaced with zero; if a component result is greater than one, it is replaced with one.

Definition 7 Inverse of an SVTNN Let $\tilde{a}=<\left(a_{1}, b_{1}, c_{1}, d_{1}\right) ; w_{\tilde{a}}, u_{\tilde{a}}, y_{\tilde{a}}>$ be an SVTNN where $a_{1}, b_{1}, c_{1}, d_{1}>0, a_{1} \leq b_{1} \leq c_{1} \leq d_{1}$, and $w_{\tilde{a}}, u_{a}, y_{\tilde{a}}, \in[0,1]$ then the inverse of $\tilde{a}$ is represented in Eq. (13):

$$
\tilde{a}^{-1}=\frac{1}{\tilde{a}}=\left\langle\left(\frac{1}{d_{1}}, \frac{1}{c_{1}}, \frac{1}{b_{1}}, \frac{1}{a_{1}}\right) ; \frac{1}{w_{\tilde{a}}}, \frac{u_{\tilde{a}}}{u_{\tilde{a}}-1}, \frac{y_{\tilde{a}}}{y_{\tilde{a}}-1}\right\rangle
$$

Remark: If a component result is less than zero, it is replaced with zero; if a component result is greater than one, it is replaced with one.

Definition 8 The TNWAA operator (Vafadarnikjoo et al., 2018). Let $\tilde{a}_{j}=<\left(a_{j}, b_{j}, c_{j}, d_{j}\right)$; $w_{\tilde{a}_{j}}, u_{\tilde{a}_{j}}, y_{\tilde{a}_{j}}>(j=1,2, \ldots, n)$ be a set of SVTNNs; then, a TNWAA operator is computed based on Eq. (14):

$$
\operatorname{TNWAA}\left(\tilde{a}_{1}, \tilde{a}_{2}, \ldots, \tilde{a}_{n}\right)=\sum_{j=1}^{n} p_{j} \tilde{a}_{j}=\left\langle\left(\sum_{j=1}^{n} p_{j} a_{j}, \sum_{j=1}^{n} p_{j} b_{j}, \sum_{j=1}^{n} p_{j} c_{j}, \sum_{j=1}^{n} p_{j} d_{j}\right) ; 1-\prod_{j=1}^{n}\left(1-w_{\tilde{a}_{j}}\right)^{p_{j}}, \prod_{j=1}^{n} u_{\tilde{a}_{j}}^{p_{j}}, \prod_{j=1}^{n} y_{\tilde{a}_{j}}^{p_{j}}\right\rangle
$$

Here, $p_{j}$ is the weight of $\tilde{a}_{j}(j=1,2, \ldots, n)$ while $p_{j}>0$, and $\sum_{j=1}^{n} p_{j}=1$.

Definition 9: Score function of a SVTNN (Vafadarnikjoo, 2020). Given $\tilde{a}=<(a, b, c, d)$; $w_{\tilde{a}}, u_{\tilde{a}}, y_{\tilde{a}}>$ and $a, b, c, d>0$. Then, the score function of $\tilde{a}$ can be calculated in accordance with Eq. (15):

$$
S(\tilde{a})=\frac{1}{12}(a+b+c+d)\left(2+w_{\tilde{a}}-u_{\tilde{a}}-y_{\tilde{a}}\right) S(\tilde{a}) \in[0,1]
$$

Acknowledgements We sincerely thank the editors and anonymous reviewers for their valuable, critical, and constructive comments, which have helped us improve our paper.

\section{References}

Abdel-Basset, M., Manogaran, G., Mohamed, M., \& Chilamkurti, N. (2018). Three-way decisions based on neutrosophic sets and AHP-QFD framework for supplier selection problem. Future Generation Computer Systems, 89, 19-30. https://doi.org/10.1016/j.future.2018.06.024.

Abeyratne, S. A., \& Monfared, R. P. (2016). Blockchain ready manufacturing supply chain using distributed ledger. International Journal of Research in Engineering and Technology, 5(9). https://dspace.lboro. ac.uk/2134/22625

Akkermans, H., Bogerd, P., \& Vos, B. (1999). Virtuous and vicious cycles on the road towards international supply chain management. International Journal of Operations \& Production Management, 19(5/6), 565-582. https://doi.org/10.1108/01443579910260883. 
Andersen, J. V., \& Ingram Bogusz, C. (2019). Self-organizing in blockchain infrastructures: Generativity through shifting objectives and forking. Journal of the Association for Information Systems. https://doi. org/https://doi.org/10.17705/1jais.00566

Angelis, J., \& Ribeiro da Silva, E. (2019). Blockchain adoption: A value driver perspective. Business Horizons, 62(3), 307-314. https://doi.org/10.1016/j.bushor.2018.12.001.

Asadi Bagloee, S., Tavana, M., Withers, G., Patriksson, M., \& Asadi, M. (2019). Tradable mobility permit with Bitcoin and Ethereum - A Blockchain application in transportation. Internet of Things, 8 , 100103. https://doi.org/10.1016/j.iot.2019.100103.

Atanassov, K. T. (1986). Intuitionistic fuzzy sets. Fuzzy Sets and Systems, 20(1), 87-96.

Ayed Mouelhi, R. B. (2009). Impact of the adoption of information and communication technologies on firm efficiency in the Tunisian manufacturing sector. Economic Modelling, 26(5), 961-967. https:// doi.org/10.1016/j.econmod.2009.03.001.

Azzi, R., Chamoun, R. K., \& Sokhn, M. (2019). The power of a blockchain-based supply chain. Computers \& Industrial Engineering, 135, 582-592. https://doi.org/10.1016/j.cie.2019.06.042.

Babich, V., \& Hilary, G. (2020). OM Forum-distributed ledgers and operations: What operations management researchers should know about blockchain technology. Manufacturing \& Service Operations Management, 22(2), 223-240. https://doi.org/10.1287/msom.2018.0752.

Badri Ahmadi, H., Kusi-Sarpong, S., \& Rezaei, J. (2017). Assessing the social sustainability of supply chains using Best Worst Method. Resources, Conservation and Recycling, 126, 99-106. https://doi. org/10.1016/j.resconrec.2017.07.020.

Bai, C., \& Sarkis, J. (2020). A supply chain transparency and sustainability technology appraisal model for blockchain technology. International Journal of Production Research, 58(7), 2142-2162. https://doi.org/10.1080/00207543.2019.1708989.

Beck, R., Müller-Bloch, C., \& King, J. L. (2018). Governance in the blockchain economy: A framework and research agenda. Journal of the Association for Information Systems, 19(10), 1020-1034.

Bender, J. P., Burchardi, K., \& Shepherd, N. (2019). Capturing the Value of Blockchain. Boston Consulting Group. https://www.bcg.com/publications/2019/capturing-blockchain-value

Biswas, B., \& Gupta, R. (2019). Analysis of barriers to implement blockchain in industry and service sectors. Computers \& Industrial Engineering, 136, 225-241. https://doi.org/10.1016/j.cie.2019.07. 005.

Bloom, N., Garicano, L., Sadun, R., \& Van Reenen, J. (2014). The distinct effects of information technology and communication technology on firm organization. Management Science, 60(12), 28592885. https://doi.org/10.1287/mnsc.2014.2013.

Bolturk, E., \& Kahraman, C. (2018). A novel interval-valued neutrosophic AHP with cosine similarity measure. Soft Computing, 22(15), 4941-4958. https://doi.org/10.1007/s00500-018-3140-y.

Büyüközkan, G., \& Göçer, F. (2018). Digital supply chain: Literature review and a proposed framework for future research. Computers in Industry, 97, 157-177. https://doi.org/10.1016/j.compind.2018. 02.010 .

Carson, B., Romanelli, G., Walsh, P., \& Zhumaev, A. (2018). Blockchain beyond the hype: What is the strategic business value? McKinsey \& Company. https://www.mckinsey.com/business-functions/ mckinsey-digital/our-insights/blockchain-beyond-the-hype-what-is-the-strategic-business-value

Chang, Y., Iakovou, E., \& Shi, W. (2020). Blockchain in global supply chains and cross border trade: A critical synthesis of the state-of-the-art, challenges and opportunities. International Journal of Production Research, 58(7), 2082-2099. https://doi.org/10.1080/00207543.2019.1651946.

Chanson, M., Bogner, A., Bilgeri, D., Fleisch, E., \& Wortmann, F. (2019). Blockchain for the IoT: Privacy-preserving protection of sensor data. Journal of the Association for Information Systems. https://doi.org/https://doi.org/10.17705/1jais.00567

Choi, T.-M. (2020). Supply chain financing using blockchain: Impacts on supply chains selling fashionable products. Annals of Operations Research. https://doi.org/10.1007/s10479-020-03615-7.

Choi, T.-M., Feng, L., \& Li, R. (2020). Information disclosure structure in supply chains with rental service platforms in the blockchain technology era. International Journal of Production Economics, 221, 107473. https://doi.org/10.1016/j.ijpe.2019.08.008.

Choi, T.-M., Guo, S., Liu, N., \& Shi, X. (2020c). Optimal pricing in on-demand-service-platform-operations with hired agents and risk-sensitive customers in the blockchain era. European Journal of Operational Research, 284(3), 1031-1042. https://doi.org/10.1016/j.ejor.2020.01.049.

Choi, T.-M., Guo, S., \& Luo, S. (2020b). When blockchain meets social-media: Will the result benefit social media analytics for supply chain operations management? Transportation Research Part E: Logistics and Transportation Review, 135, 101860. https://doi.org/10.1016/j.tre.2020.101860. 
Choi, T.-M., Wen, X., Sun, X., \& Chung, S.-H. (2019). The mean-variance approach for global supply chain risk analysis with air logistics in the blockchain technology era. Transportation Research Part E: Logistics and Transportation Review, 127, 178-191. https://doi.org/10.1016/j.tre.2019.05.007.

Chong, A. Y. L., Lim, E. T. K., Hua, X., Zheng, S., \& Tan, C.-W. (2019). Business on chain: A comparative case study of five blockchain-inspired business models. Journal of the Association for Information Systems. https://doi.org/https://doi.org/10.17705/1jais.00568

Cohen, S., \& Zohar, A. (2018). Database Perspectives on Blockchains.

Cole, R., Stevenson, M., \& Aitken, J. (2019). Blockchain technology: Implications for operations and supply chain management. Supply Chain Management: An International Journal, 24(4), 469-483. https://doi. org/10.1108/SCM-09-2018-0309.

De Giovanni, P. (2020). Blockchain and smart contracts in supply chain management: A game theoretic model. International Journal of Production Economics, 228, 107855. https://doi.org/10.1016/j.ijpe. 2020.107855.

Deli, I., \& Subas, Y. (2014). Single valued neutrosophic numbers and their applications to multicriteria decision making problem. Neutrosophic Sets and Systems, 2(1), 1-13.

Dolgui, A., Ivanov, D., Potryasaev, S., Sokolov, B., Ivanova, M., \& Werner, F. (2020). Blockchain-oriented dynamic modelling of smart contract design and execution in the supply chain. International Journal of Production Research, 58(7), 2184-2199. https://doi.org/10.1080/00207543.2019.1627439.

Dutta, P., Choi, T.-M., Somani, S., \& Butala, R. (2020). Blockchain technology in supply chain operations: Applications, challenges and research opportunities. Transportation Research Part E: Logistics and Transportation Review, 142, 102067. https://doi.org/10.1016/j.tre.2020.102067.

Ecer, F. (2020). Multi-criteria decision making for green supplier selection using interval type-2 fuzzy AHP: A case study of a home appliance manufacturer. Operational Research. https://doi.org/10.1007/ s12351-020-00552-y.

Emrouznejad, A., \& Ho, W. (Eds.). (2017). Fuzzy analytic hierarchy process. . CRC Press.

Emrouznejad, A., \& Marra, M. (2017). The state of the art development of AHP (1979-2017): A literature review with a social network analysis. International Journal of Production Research, 55(22), 66536675. https://doi.org/10.1080/00207543.2017.1334976.

Esmaeilian, B., Sarkis, J., Lewis, K., \& Behdad, S. (2020). Blockchain for the future of sustainable supply chain management in Industry 4.0. Resources, Conservation and Recycling. https://doi.org/10.1016/j. resconrec.2020.105064.

Fernandez-Carames, T. M., \& Fraga-Lamas, P. (2019). A review on the application of blockchain to the next generation of cybersecure industry 4.0 smart factories. IEEE Access, 7, 45201-45218. https://doi.org/ 10.1109/ACCESS.2019.2908780.

Fosso Wamba, S., Kala Kamdjoug, J. R., Epie Bawack, R., \& Keogh, J. G. (2020). Bitcoin, Blockchain and Fintech: A systematic review and case studies in the supply chain. Production Planning \& Control, 31(2-3), 115-142. https://doi.org/10.1080/09537287.2019.1631460.

Frizzo-Barker, J., Chow-White, P. A., Adams, P. R., Mentanko, J., Ha, D., \& Green, S. (2020). Blockchain as a disruptive technology for business: A systematic review. International Journal of Information Management, 51, 102029. https://doi.org/10.1016/j.ijinfomgt.2019.10.014.

Ganeriwalla, A., Casey, M., Shrikrishna, P., Bender, J. P., \& Gstettner, S. (2018). Does your supply chain need a blockchain? Boston Consulting Group. https://www.bcg.com/en-gb/publications/2018/doesyour-supply-chain-need-blockchain

Ghode, D., Yadav, V., Jain, R., \& Soni, G. (2020). Adoption of blockchain in supply chain: An analysis of influencing factors. Journal of Enterprise Management, Information ahead-of-print(ahead-of-print). https://doi.org/10.1108/JEIM-07-2019-0186.

Grant, D., \& Yeo, B. (2018). A global perspective on tech investment, financing, and ICT on manufacturing and service industry performance. International Journal of Information Management, 43, 130-145. https://doi.org/10.1016/j.ijinfomgt.2018.06.007.

Hastig, G. M., \& Sodhi, M. S. (2020). Blockchain for Supply chain traceability: Business requirements and critical success factors. Production and Operations Management, poms. https://doi.org/10.1111/poms. 13147.

Hayaty, M., Tavakoli Mohammadi, M. R., Rezaei, A., \& Shayestehfar, M. R. (2014). Risk assessment and ranking of metals using FDAHP and TOPSIS. Mine Water and the Environment, 33(2), 157-164. https://doi.org/10.1007/s10230-014-0263-y.

Helo, P., \& Hao, Y. (2019). Blockchains in operations and supply chains: A model and reference implementation. Computers \& Industrial Engineering, 136, 242-251. https://doi.org/10.1016/j.cie.2019.07.023.

Iansiti, M., \& Lakhani, K. R. (2017). The truth about blockchain. Harvard Business Review, 95(1), 118-127. 
Ivanov, D., Dolgui, A., \& Sokolov, B. (2019). The impact of digital technology and Industry 4.0 on the ripple effect and supply chain risk analytics. International Journal of Production Research, 57(3), 829-846.

Jabbar, A., \& Dani, S. (2020). Investigating the link between transaction and computational costs in a blockchain environment. International Journal of Production Research, 58(11), 3423-3436. https://doi.org/ 10.1080/00207543.2020.1754487.

Jeffers, P. I. (2010). Embracing sustainability: Information technology and the strategic leveraging of operations in third-party logistics. International Journal of Operations \& Production Management, 30(3), 260-287. https://doi.org/10.1108/01443571011024629.

Kane, E. (2017). Is Blockchain a general purpose technology? SSRN Electronic Journal. https://doi.org/10. 2139/ssrn.2932585.

Kayikci, Y., Subramanian, N., Dora, M., \& Bhatia, M. S. (2020). Food supply chain in the era of Industry 4.0: Blockchain technology implementation opportunities and impediments from the perspective of people, process, performance, and technology. Production Planning \& Control. https://doi.org/10.1080/09537287. 2020.1810757.

Kouhizadeh, M., Saberi, S., \& Sarkis, J. (2021). Blockchain technology and the sustainable supply chain: Theoretically exploring adoption barriers. International Journal of Production Economics, 231, 107831. https://doi.org/10.1016/j.ijpe.2020.107831.

Kouhizadeh, M., \& Sarkis, J. (2018). Blockchain practices, potentials, and perspectives in greening supply chains. Sustainability, 10(10), 3652. https://doi.org/10.3390/su10103652.

Kouhizadeh, M., Sarkis, J., \& Zhu, Q. (2019). At the nexus of blockchain technology, the circular economy, and product deletion. Applied Sciences, 9(8), 1712. https://doi.org/10.3390/app9081712.

Kouhizadeh, M., Zhu, Q., \& Sarkis, J. (2019). Blockchain and the circular economy: Potential tensions and critical reflections from practice. Production Planning \& Control. https://doi.org/10.1080/09537287.2019. 1695925.

Lee, S. M., Lee, D., \& Schniederjans, M. J. (2011). Supply chain innovation and organizational performance in the healthcare industry. International Journal of Operations \& Production Management, 31(11), 11931214. https://doi.org/10.1108/01443571111178493.

Lim, M. K., Li, Y., Wang, C., \& Tseng, M.-L. (2021). A literature review of blockchain technology applications in supply chains: A comprehensive analysis of themes, methodologies and industries. Computers \& Industrial Engineering, 154, 107133. https://doi.org/10.1016/j.cie.2021.107133.

Lin, Y.-P., Petway, J., Anthony, J., Mukhtar, H., Liao, S.-W., Chou, C.-F., \& Ho, Y.-F. (2017). Blockchain: The evolutionary next step for ICT E-agriculture. Environments, 4(3), 50. https://doi.org/10.3390/environmen ts4030050.

Lohmer, J., Bugert, N., \& Lasch, R. (2020). Analysis of resilience strategies and ripple effect in blockchaincoordinated supply chains: An agent-based simulation study. International Journal of Production Economics, 228, 107882. https://doi.org/10.1016/j.ijpe.2020.107882.

Longo, F., Nicoletti, L., Padovano, A., d'Atri, G., \& Forte, M. (2019). Blockchain-enabled supply chain: An experimental study. Computers \& Industrial Engineering, 136, 57-69. https://doi.org/10.1016/j.cie.2019. 07.026.

Luthra, S., Kumar, A., Zavadskas, E. K., Mangla, S. K., \& Garza-Reyes, J. A. (2020). Industry 4.0 as an enabler of sustainability diffusion in supply chain: An analysis of influential strength of drivers in an emerging economy. International Journal of Production Research, 58(5), 1505-1521.

Manupati, V. K., Schoenherr, T., Ramkumar, M., Wagner, S. M., Pabba, S. K., \& Singh, I. R. (2020). A blockchain-based approach for a multi-echelon sustainable supply chain. International Journal of Production Research, 58(7), 2222-2241. https://doi.org/10.1080/00207543.2019.1683248.

Moghadam, A. H., \& Assar, P. (2008). The relationship between national culture and E-adoption: A case study of Iran. American Journal of Applied Sciences, 5(4), 369-377. https://doi.org/10.3844/ajassp.2008.369. 377.

Moser, M., Bohme, R., \& Breuker, D. (2013). An inquiry into money laundering tools in the Bitcoin ecosystem. APWG ECrime Researchers Summit, 2013, 1-14. https://doi.org/10.1109/eCRS.2013.6805780.

Petersen, M., Hackius, N., \& von See, B. (2018). Mapping the sea of opportunities: Blockchain in supply chain and logistics. It - Information Technology, 60(5-6), 263-271. https://doi.org/10.1515/itit-2017-0031.

Pournader, M., Shi, Y., Seuring, S., \& Koh, S. C. L. (2020). Blockchain applications in supply chains, transport and logistics: A systematic review of the literature. International Journal of Production Research, 58(7), 2063-2081. https://doi.org/10.1080/00207543.2019.1650976.

Rahmanzadeh, S., Pishvaee, M. S., \& Rasouli, M. R. (2020). Integrated innovative product design and supply chain tactical planning within a blockchain platform. International Journal of Production Research, 58(7), 2242-2262. https://doi.org/10.1080/00207543.2019.1651947. 
Risius, M., \& Spohrer, K. (2017). A blockchain research framework: What we (don't) know, where we go from here, and how we will get there. Business \& Information Systems Engineering, 59(6), 385-409. https://doi. org/10.1007/s12599-017-0506-0.

Rossi, M., Mueller-Bloch, C., Thatcher, J. B., \& Beck, R. (2019). Blockchain research in information systems: Current trends and an inclusive future research agenda. Journal of the Association for Information Systems. https://doi.org/https://doi.org/10.17705/1jais.00571

Saaty, T. L. (1980). The analytic hierarchy process: Planning, priority setting, resource allocation. McGrawHill International Book Co.

Saberi, S., Kouhizadeh, M., \& Sarkis, J. (2018). Blockchain technology: A panacea or pariah for resources conservation and recycling? Resources, Conservation and Recycling, 130, 80-81. https://doi.org/10.1016/j. resconrec.2017.11.020.

Saberi, S., Kouhizadeh, M., \& Sarkis, J. (2019a). Blockchains and the supply chain: Findings from a broad study of practitioners. IEEE Engineering Management Review, 47(3), 95-103. https://doi.org/10.1109/ EMR.2019.2928264.

Saberi, S., Kouhizadeh, M., Sarkis, J., \& Shen, L. (2019b). Blockchain technology and its relationships to sustainable supply chain management. International Journal of Production Research, 57(7), 2117-2135. https://doi.org/10.1080/00207543.2018.1533261.

Sharif, A. M., Irani, Z., \& Lloyd, D. (2007). Information technology and performance management for build-toorder supply chains. International Journal of Operations \& Production Management, 27(11), 1235-1253. https://doi.org/10.1108/01443570710830610.

Sitorus, F., Cilliers, J. J., \& Brito-Parada, P. R. (2019). An integrated constrained fuzzy stochastic analytic hierarchy process method with application to the choice problem. Expert Systems with Applications, 138, 112822. https://doi.org/10.1016/j.eswa.2019.112822.

Smarandache, F. (2005). Neutrosophic set-a generalization of the intuitionistic fuzzy set. International Journal of Pure and Applied Mathematics, 24(3), 287-297.

Smarandache, F. (2016). Subtraction and division of neutrosophic numbers. Critical Review: A Publication of Society for Mathematics of Uncertainty, 13, 103-110.

Swan, M. (2015). Blockchain: Blueprint for a new economy (First edition). O'Reilly.

Vafadarnikjoo, A. (2020). Decision analysis in the UK energy supply chain risk management: Tools development and application. https://ueaeprints.uea.ac.uk/id/eprint/77909

Vafadarnikjoo, A., Mishra, N., Govindan, K., \& Chalvatzis, K. (2018). Assessment of consumers' motivations to purchase a remanufactured product by applying Fuzzy Delphi method and single valued neutrosophic sets. Journal of Cleaner Production, 196, 230-244. https://doi.org/10.1016/j.jclepro.2018.06.037.

Vafadarnikjoo, A., Tavana, M., Botelho, T., \& Chalvatzis, K. (2020). A neutrosophic enhanced best-worst method for considering decision-makers' confidence in the best and worst criteria. Annals of Operations Research, 289, 391-418. https://doi.org/10.1007/s10479-020-03603-x.

Vatankhah Barenji, A., Li, Z., Wang, W. M., Huang, G. Q., \& Guerra-Zubiaga, D. A. (2020). Blockchain-based ubiquitous manufacturing: A secure and reliable cyber-physical system. International Journal of Production Research, 58(7), 2200-2221. https://doi.org/10.1080/00207543.2019.1680899.

Vukolić, M. (2016). The quest for scalable Blockchain fabric: Proof-of-work vs. BFT replication. In J. Camenisch \& D. Kesdoğan (Eds.), Open problems in network security. (pp. 112-125). Springer.

Wamba, S. F., \& Queiroz, M. M. (2020). Industry 4.0 and the supply chain digitalisation: A blockchain diffusion perspective. Production Planning \& Control. https://doi.org/10.1080/09537287.2020.1810756.

Wang, Y., Han, J. H., \& Beynon-Davies, P. (2019). Understanding blockchain technology for future supply chains: A systematic literature review and research agenda. Supply Chain Management: An International Journal, 24(1), 62-84. https://doi.org/10.1108/SCM-03-2018-0148.

Wang, Y., Singgih, M., Wang, J., \& Rit, M. (2019). Making sense of blockchain technology: How will it transform supply chains? International Journal of Production Economics, 211, 221-236. https://doi.org/10. 1016/j.ijpe.2019.02.002.

White, G. R. T. (2017). Future applications of blockchain in business and management: A Delphi study. Strategic Change, 26(5), 439-451. https://doi.org/10.1002/jsc.2144.

Wong, L.-W., Tan, G.W.-H., Lee, V.-H., Ooi, K.-B., \& Sohal, A. (2020). Unearthing the determinants of Blockchain adoption in supply chain management. International Journal of Production Research, 58(7), 21002123. https://doi.org/10.1080/00207543.2020.1730463.

Xie, J., Yu, F. R., Huang, T., Xie, R., Liu, J., \& Liu, Y. (2019). A Survey on the scalability of Blockchain systems. IEEE Network, 33(5), 166-173. https://doi.org/10.1109/MNET.001.1800290.

Yli-Huumo, J., Ko, D., Choi, S., Park, S., \& Smolander, K. (2016). Where Is current research on Blockchain technology? -A systematic review. PLoS ONE, 11(10), e0163477. https://doi.org/10.1371/journal.pone. 0163477. 
Yoon, J., Talluri, S., Yildiz, H., \& Sheu, C. (2020). The value of Blockchain technology implementation in international trades under demand volatility risk. International Journal of Production Research, 58(7), 2163-2183. https://doi.org/10.1080/00207543.2019.1693651.

Zhang, R., Xue, R., \& Liu, L. (2019). Security and privacy on Blockchain. ACM Computing Surveys, 52(3), 1-34. https://doi.org/10.1145/3316481.

Publisher's Note Springer Nature remains neutral with regard to jurisdictional claims in published maps and institutional affiliations. 